Review Article

\title{
Self-Organization Schemes towards Thermodynamic Stable Bulk Heterojunction Morphologies: A Perspective on Future Fabrication Strategies of Polymer Photovoltaic Architectures
}

\author{
A. Benmouna, ${ }^{1}$ R. Benmouna, ${ }^{1}$ M. R. Bockstaller, ${ }^{2}$ and I. F. Hakem ${ }^{2}$ \\ ${ }^{1}$ Department of Physics, Faculty of Sciences, University of Abou Bekr Belkaid, Tlemcen, Algeria \\ ${ }^{2}$ Department of Materials Science and Engineering, Carnegie Mellon University, 5000 Forbes Avenue, Pittsburgh, PA 15243, USA
}

Correspondence should be addressed to I. F. Hakem; hakemif@cmu.edu

Received 26 November 2012; Revised 26 March 2013; Accepted 27 March 2013

Academic Editor: Francesco Paolucci

Copyright (c) 2013 A. Benmouna et al. This is an open access article distributed under the Creative Commons Attribution License, which permits unrestricted use, distribution, and reproduction in any medium, provided the original work is properly cited.

\begin{abstract}
Research efforts to improve our understanding of electronic polymers are developing fast because of their promising advantages over silicon in photovoltaic solar cells. A major challenge in the development of polymer photovoltaic devices is the viable fabrication strategies of stable bulk heterojunction architecture that will retain functionality during the expected lifetime of the device. Block copolymer self-assembly strategies have attracted particular attention as a scalable means toward thermodynamically stable microstructures that combine the ideal geometrical characteristics of a bulk heterojunction with the fortuitous combination of properties of the constituent blocks. Two primary routes that have been proposed in the literature involve the coassembly of block copolymers in which one domain is a hole conductor with the electron-conducting filler (such as fullerene derivatives) or the selfassembly of block copolymers in which the respective blocks function as hole and electron conductor. Either way has proven difficult because of the combination of synthetic challenges as well as the missing understanding of the complex governing parameters that control structure formation in semiconducting block copolymer blends. This paper summarizes important findings relating to structure formation of block copolymer and block copolymer/nanoparticle blend assembly that should provide a foundation for the future design of block copolymer-based photovoltaic systems.
\end{abstract}

\section{Introduction}

Solar cells from silicon ( $\mathrm{Si}$ ) represent the essential part of the solar photovoltaic (PV) cells on the market. But the Si technology together with other metal composites like CdTe and GaAs has major drawbacks such as high process cost, hazard to the environment, and pollution in addition to a limited availability $[1,2]$. Polymer-based PV cells can be viewed as an alternative or at least a complement to those standard technologies [3-5]. The active layer of the polymer composite is easy to process and can be deposited on a solid surface from a solution. The process cost is drastically reduced compared to standard inorganic cells, and large surface areas can be obtained. The cells are flexible with a shape designed almost at will according to the needs but nevertheless have a strong mechanical resistance [6-8]. The main challenge that remains to be faced is the possibility of enhancing the efficiency $[8,9]$ which is continuously improving by using the appropriate polymer system with the right architecture and morphology $[9,10]$.

The active polymer layer collects the incident light from the sun and converts it into excitons or bound electrons/holes pairs. Those are then dissociated into free charge carriers before flowing to the electrodes to produce an electric current $[11,12]$. The quantum efficiency defines the polymers' capability to convert photons into charge carriers that are subsequently transported by one of two different mechanisms, that is, by transporting one along chains or hopping between distinct chains. Delocalization and diffusion of charge carriers along chains are hindered by defects in their structure and perturbations due to the presence of host molecules or charges in the environment. Interchain conformation and morphology considerations affect the hopping process, and chains often exhibit a certain degree of disorder that leads to 
a difficulty in determining the HOMO and LUMO levels in the presence of a random distribution of hopping sites [13, 14].

Morphologies based on nanostructured donor/acceptor constituents have attracted a lot of interest because of the ideal contact between the electron donor (D) conjugated polymer and the electron acceptor (A) component. A large D to A contact area favors exciton collection and dissociation into free electron and hole carriers, while a large number of transport pathways allow free charges to flow into the electrodes. Continued developments are being reported in the literature by using conjugated interpenetrated polymer networks, block copolymers, and other polymer composites containing essentially fullerene (and its derivatives) as the electron-conducting phase. These systems put together the electrons donor and acceptor to favor exciton dissociation and charge transport and are regarded as a major alternative to the Si technology. Research efforts nowadays combine both the improvement of optoelectronic properties of the polymer material and its morphology [15-17].

Fullerene and its derivatives are currently among the best electron acceptors with high efficiency. They are characterized by a high solubility in standard solvents and allow processing under wet conditions. A typical example is given by the blend of poly(3-hexylthiophene) (P3HT) and 1-[3-(methoxycarbonyl)propyl]-1-phenyl-[6, 6] C61 (PCBM) which is among the most widely used in the literature [1820]. These chemical species are commercially available and provide a reference for polymer-based photovoltaic cells.

The concomitance of D/A components reminiscent of the copolymer structure permits to optimize losses in the diffusion and dissociation of excitons, providing pathways for charge transport to the electrodes. The ability to control the conditions of synthesis and design the adequate morphology in terms of nanoassembled crystalline structures with a long standing stability is the way to meet challenges making the copolymer-based photovoltaic technology more competitive. An important aspect to keep in mind in developing those devices is to insure long-term operation by improving the aging properties. The equilibrium nature of diblock copolymer microstructures provides good conditions for alleviating the problems of physical aging.

This paper is organized into two parts. First, we review recent work done in the area of block copolymer/nanoparticle composites in order to provide the context that forms the basis for the proposed assembly of thermodynamically stable bulk heterojunction materials. Second, we summarize specific features of conducting block copolymer systems that give rise to a wider parameter range and scientific challenges that need to be addressed.

\section{Block Copolymer Self-Assembly as Means towards Tuning the Morphology of the Polymer Medium}

Controlling the microstructure of bulk heterojunction morphologies in order to minimize exciton diffusion pathways is of critical importance in realizing efficient and economically viable device architectures. Current material systems

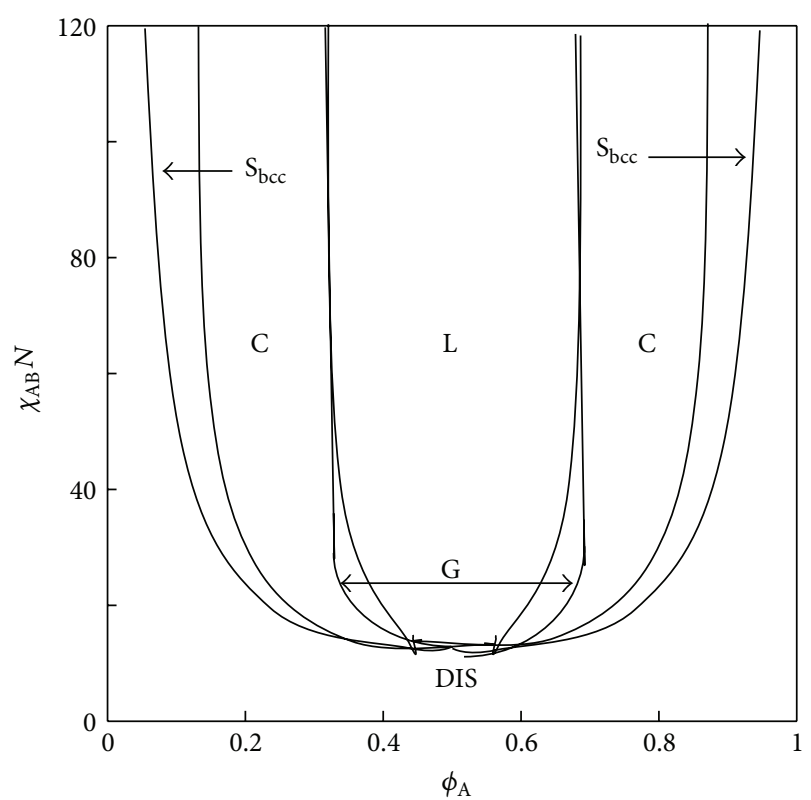

Figure 1: Phase diagram of a diblock copolymer. The vertical axis is a weighted interaction parameter $\left(\chi_{\mathrm{AB}} N\right)$, and the horizontal axis is the volume fraction of polymer $\mathrm{A}\left(\phi_{\mathrm{A}}\right)$. Each phase is identified by a letter representing the structure: $\mathrm{L}$-lamellae, $\mathrm{G}$ - gyroid, $\mathrm{C}-$ cylinder, and S-sphere [24].

based on blends of conducting polymer and PCBM rely on the formation of bicontinuous network morphologies during the spinodal decomposition of the polymer/PCBM blend to realize characteristic length scales of about $10 \mathrm{~nm}$ between the electron and hole-conducting phase. A principal disadvantage of this approach is the inherent thermodynamic instability of the resulting microstructure that gives rise to rapid physical aging and performance degradation if devices are operated at temperatures above $50^{\circ} \mathrm{C}$.

The challenges associated with the instability of kinetically trapped heterojunctions has fueled interest in an alternative approach to bulk heterojunction fabrication that is based on the formation of thermodynamic stable microdomain morphologies in block copolymer systems. Block copolymers are macromolecules that combine two or more polymer chains via covalent bonding. They have attracted widespread interest because of their ability to self-organize into a hierarchy of periodic microdomain morphologies [21]. This self-organization is driven by the tendency of phase separation of the individual chains thatbecause of the constraint of the covalent junction-form frustrated microdomain structures. The type of structure being formed depends on the interplay of two competing interactions: the surface tension between the domains formed by the respective blocks and the conformational entropy of the polymer chains. The balance of these interactions gives rise to the famous "Block Copolymer Phase Diagram" that was first postulated by Leibler for weakly interacting systems and later generalized by Bates and Fredrickson [22, 23]. Block copolymers are differentiated by the number and connectivity of blocks present in their structure, as shown in Figure 1. 
The diblock copolymer structure that is the focus of this study consists of $\mathrm{A} / \mathrm{B}$ type monomers (in this case, styrene and methyl methacrylate) that self-assemble into distinctive morphologies. The phase behavior of block copolymers depends upon the degree of polymerization $(N)$, the structure (diblock, triblock, or starblock), the volume fraction of each component $(\phi)$, and the Flory-Huggins interaction parameter $\left(\chi_{\mathrm{AB}}\right)$ [23]. Thermodynamic considerations result in minimum free energy configurations, as shown in the phase diagram in Figure 1 [24]. The classical BCP phase diagram differentiates four distinct structures that are expected to form depending on the composition and the degree of segregation of the BCP. These morphologies (lamellae, gyroids, cylinders, and spheres) are indicated on the phase diagram and are depicted in the schematic in Figure 1 [22-25]. Two major strategies have been described in the literature to take advantage of block copolymer self-assembly for the fabrication of bulk heterojunction structures: (1) the coassembly of a semiconducting-insulating $\mathrm{AB}$ diblock copolymer (in which block A represents the hole-conducting block such as P3HT and block B represents an insulating block such as polystyrene, PS) admixed with electron-conducting PCBMtype filler. This approach is based on the idea that the filler-if appropriately surface modified-will sequester at the interface between both domains and form an interconnected electron-conducting path. In principle this approach should provide an ideal solution for the fabrication of improved bulk heterojunction morphologies because it facilitates the combination of efficient hole and electron-conducting systems (such as P3HT and PCBM), with advantageous properties of the nonactive second block (that could, e.g., reduce oxygen permeation and thus increase the lifetime of the device) with the thermodynamic stability and geometry of the microphase-separated structure. Unfortunately, realization of this approach has proven very difficult due to the complex nature of the structure formation process in these multicomponent systems. Here, insights gained by research on amorphous block copolymer/nanoparticle blend systems (described in Section 3) could provide a helpful resource to select material and process conditions that are favorable for suitable structure formation. (2) A second approach that has been perpetrated by several groups is the combination of electron and hole-conducting blocks in block copolymer architectures and the subsequent assembly of the copolymer in microdomain morphologies. This approach must generally be seen as more challenging because of the complicated synthesis of all-semiconducting block copolymer systems (especially with the required small polydispersity to facilitate microphase separation) and the absence of suitable polymer compositions with comparable electron transport characteristics and energy level lineup as PCBM. Considering the fact that conjugated polymers $[26,27]$ are rod-like, a further challenge is provided by the insufficient understanding of the structure formation in rod-like block copolymers that generally defy the simple phase diagram depicted in Figure 1.

In order to facilitate further progress in the fabrication of thermodynamic stable bulk heterojunction materials research will have to accomplish better understanding of the structure formation processes characteristic to the systems outlined above. A foundation for future research is provided by the understanding that has been obtained in related fields of polymer science such as amorphous block copolymer/nanoparticle blends as well as (nonconducive) rod-coil type block copolymer materials. In the following some of the major conclusions regarding the governing parameters for structure formation in these systems will be summarized. Since the major trends of structure formation are expected to be universal across the different classes of polymer materials, these conclusions should inform future research in the area of bulk heterojunction materials.

\section{Block Copolymer/Nanoparticle Blend Materials}

3.1. Structure Formation in Block Copolymer/Nanoparticle Blends: Experiment and Simulation. A significant amount of work has been done on $\mathrm{BCP} /$ nanoparticle blend systems since they offer a wealth of opportunities to combine the properties of both the filler nanoparticle and the host polymer. The result is a composite material with enhanced mechanical, optical, or thermal properties that maintains the flexibility, optical transparency, or other features of the original polymer matrix.

In 2001, Thompson et al. [28] combined density functional theoretical and self-consistent field theoretical computer simulations to examine nanoparticle dispersion in a lamellar block copolymer system. It was found that for small particles $(d / L=0.2$ where $d$ denotes the particle diameter and $L$ the lamellar period), segregation at the intermaterial dividing surface (IMDS) occurred, while, for large particles $(d / L=0.3)$, center segregation in the compatible domain occurred. This difference in dispersion behavior of nanoparticles depending on particle size can be explained by the balance between the conformational entropy penalty of the BCP's accommodation of particles in a domain and the translational entropy gain of the freely dispersed nanoparticles [28].

In the early 2000s, Bockstaller and Thomas performed experiments that supported these computational findings. Copolymers of poly(styrene- $b$-ethylenepropylene) (PS-PEP) with $\sim 3 \mathrm{~nm}$ diameter aliphatic coated gold nanocrystals (AuSC ${ }_{12} \mathrm{H}_{25}$ ) nanoparticles were compared with PS-b-PEP with $\sim 22 \mathrm{~nm}$ diameter $\mathrm{SiO} 2-\left(\mathrm{C}_{4} \mathrm{H}_{9}\right)$ nanoparticles. The small gold nanoparticles dispersed along the IMDS, while the large silica nanoparticles segregated to the middle of the PEP domain (compatible with the aliphatic ligands on the particles), as shown in Figures 2(a) and 2(b). Note that the particle assembly along the microdomain interfaces that is shown in the upper panel would be of outmost interest as candidate structure for bulk heterojunction design if it could be realized for suitable semiconducting block copolymer/PCBM compositions.

Given the similar enthalpic contributions for both material systems depicted in Figure 2, the authors attributed the difference in particle dispersion to the competition between entropic contribution associated with the particle 


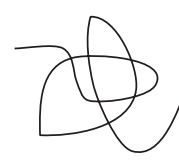

PS

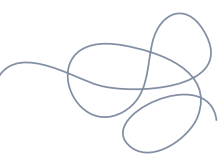

PEP
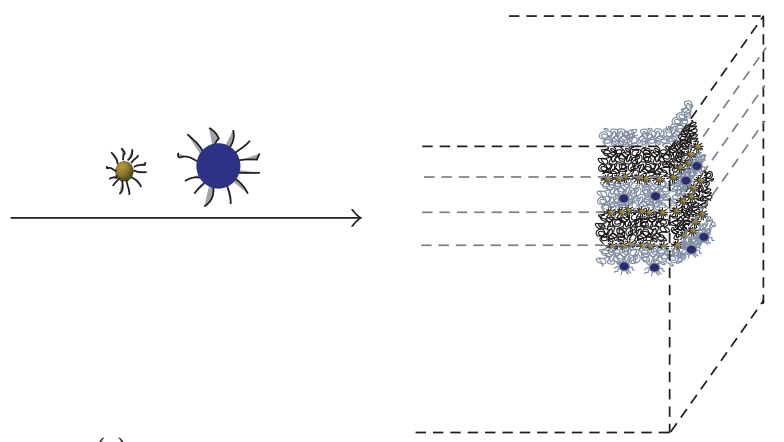

(a)

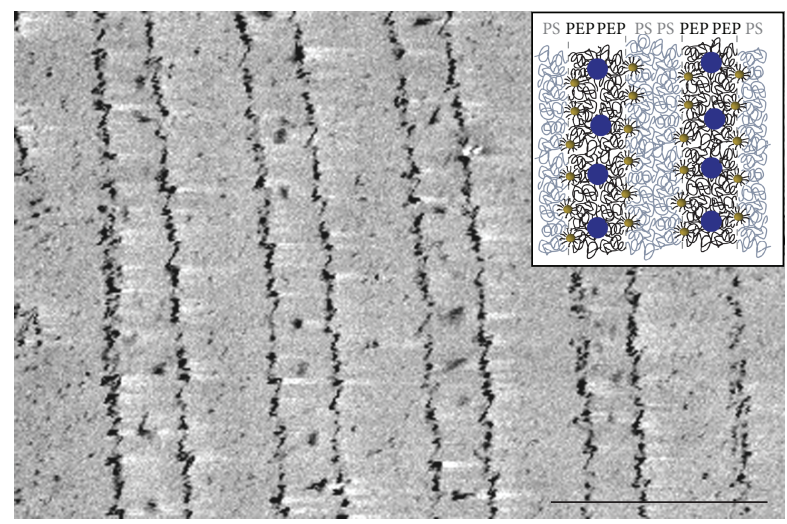

(b)

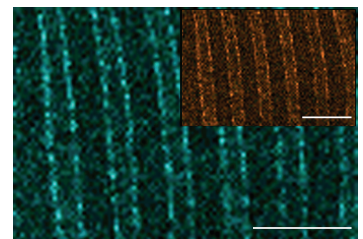

(c)

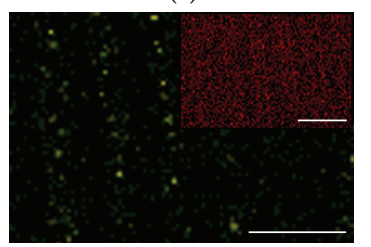

(d)

FIGURE 2: (a) TEM micrograph and schematic of PS-b-PEP/AuSC12H25 depicting the nanofillers distributed at the boundary between the PEP and PS. (b) TEM micrograph and schematic of PS-b-PEP/SiO $-\left(\mathrm{C}_{3} \mathrm{H}_{9}\right)$ depicting the nanofillers distributed in the middle of the PEP domain [21].

configurational and polymer chain conformation entropy [21]. Similar segregation behavior has also been observed for related BCP/homopolymer blends by Tanaka et al. [29] in block copolymer/homopolymer blend systems. For example, in case of a blend of poly (styrene-b-isoprene) (PS-PI) with homo-PS it was reported that for low molecular weight PS dispersion occurred throughout the PS domain and near the IMDS. Higher molecular weight PS was limited to center segregation in the PS domain, while extremely high molecular weight hPS experienced aggregation and macrophase separation [29]. In the same year, Winey et al. reported a systematic study of PS-PI/PS with varied PS molecular weight and concentration. At a fixed molecular weight, as concentration increased, the difference between dispersion in low and that in high molecular weight PS was identified by SAXS. High molecular weight PS segregated to the center of the PS domain, increasing the lamellar spacing of the PS domain without affecting the PI domain. Low molecular weight PS dispersed uniformly in the PS domain, causing the PS domain spacing to increase and the PI domain spacing to decrease [30].

The effect of enthalpic interactions has been studied by Chiu et al. [31] who analyzed structure formation in blends of PS-P2VP with gold nanoparticles modified with PS ligands. Upon variation of grafting density, the particles with a high grafting density of PS segregated to the center of the PS domain (favorable enthalpic interactions), while the particles with a low grafting density of PS experienced only partial shielding and dispersed along the IMDS (neutral enthalpic interactions) [31]. Finally, Listak et al. [32] evaluated the effect of particle size and polymer connectivity on the segregation of particle fillers and observed both center segregated and uniform particle distributions for large and small particle fillers, respectively. The authors also observed that both morphological states could be distinguished by the effect of particle addition on the characteristic domain spacing of the copolymer.

\subsection{Theoretical Modeling of Microphase Separation in BCP/NP} Blends. Morphology, phase behavior, and structural properties are intimately correlated in the polymer blend forming the active layer of the photovoltaic cell. These properties can be probed by a variety of complementary techniques and are the subject of intense research efforts including theoretical modeling [33-35]. For a polymer blend of A and B species the phase behavior is governed by the Flory-Huggins [36] interaction parameter $\chi_{\mathrm{AB}}$ which is directly related to the temperature volume fractions $\phi_{\mathrm{A}}=\phi$ and $\phi_{\mathrm{B}}=1-\phi$ and degrees of polymerization $N_{\mathrm{A}}$ and $N_{\mathrm{B}}$ :

$$
\frac{f}{k_{\mathrm{B}} T}=\frac{\phi_{\mathrm{A}}}{N_{\mathrm{A}}} \ln \phi_{\mathrm{A}}+\frac{\phi_{\mathrm{B}}}{N_{\mathrm{B}}} \ln \phi_{\mathrm{B}}+\chi_{\mathrm{AB}} \phi_{\mathrm{A}} \phi_{\mathrm{B}} .
$$




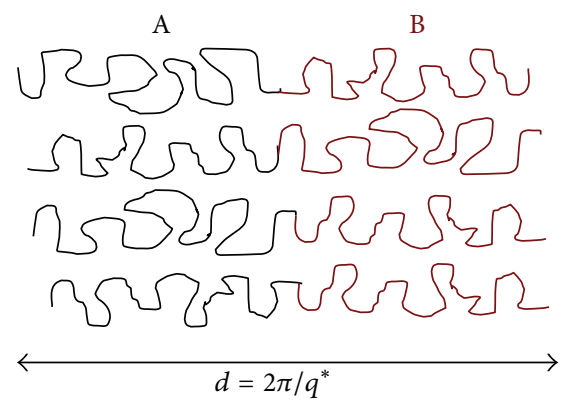

(a)

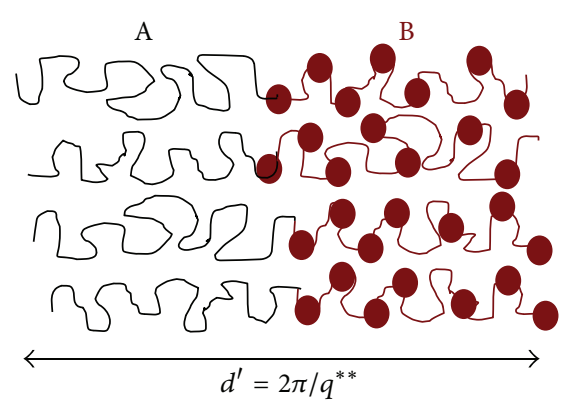

(b)

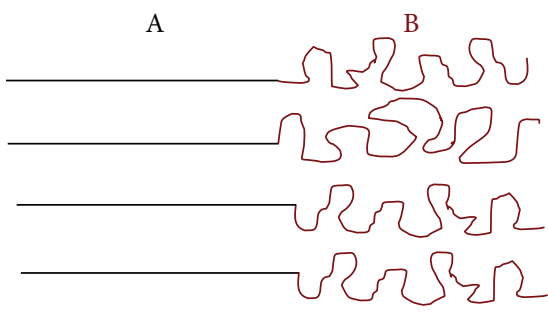

(c)

FIGURE 3: Schematic representation of diblock copolymers: (a) coil-coil, (b) with nanoparticles, and (c) coil-rod.

The blend phase separates via spinodal decomposition when $\chi_{\mathrm{AB}}$ exceeds $\chi_{S}$

$$
\chi_{S}=\frac{1}{2}\left[\frac{1}{N_{\mathrm{A}} \phi_{\mathrm{A}}}+\frac{1}{N_{\mathrm{B}} \phi_{\mathrm{B}}}\right] .
$$

For a blend with an upper critical solution temperature (UCST), if the temperature is below $T_{S}$, the system phase separates (via macrophase separation) and exhibits two distinct phases with different compositions. However, if the two polymer species are covalently bound as in a diblock copolymer, the picture changes completely and the system exhibits a microphase separation with a characteristic length $d \equiv 2 \pi / q^{*}$, where $q^{*}$ is the wavelength at which the scattering function $S(q)$ admits a maximum [22]:

$$
S^{-1}(q)=\frac{S_{0 T}(q)}{S_{0 \mathrm{~A}}(q) S_{0 \mathrm{~B}}(q)-S_{0 \mathrm{AB}}^{2}(q)}-2 \chi_{\mathrm{AB}},
$$

where

$$
\begin{gathered}
S_{0 \mathrm{~A}}=\phi_{\mathrm{A}} N_{\mathrm{A}} P_{\mathrm{A}}(q), \quad S_{0 \mathrm{~B}}=\phi_{\mathrm{B}} N_{\mathrm{B}} P_{\mathrm{B}}(q), \\
S_{0 \mathrm{AB}}=\sqrt{\phi_{\mathrm{A}} \phi_{\mathrm{B}} N_{\mathrm{A}} N_{\mathrm{B}}} P_{\mathrm{AB}}(q), \\
S_{0 T}(q)=S_{0 \mathrm{~A}}(q)+S_{0 \mathrm{~B}}(q)+2 S_{0 \mathrm{AB}}(q) .
\end{gathered}
$$

The form factors $P_{\mathrm{A}}(q), P_{\mathrm{B}}(q)$, and $P_{T}(q)$ are given by the celebrated Debye function [37]:

$$
P_{\mathrm{A}}(q)=\frac{2}{u_{\mathrm{A}}^{2}}\left[\exp \left(-u_{\mathrm{A}}\right)+u_{\mathrm{A}}-1\right] ; \quad u_{\mathrm{A}}=q^{2} R_{\mathrm{gA}}^{2}
$$

$R_{\mathrm{gA}}$ being the radius of gyration of block $\mathrm{A}$, that is, $R_{\mathrm{gA}}^{2} \equiv$ $N_{A} l^{2} / 6$, and $l$ is the length of a monomer. Similar expressions hold for $P_{\mathrm{B}}(q)$ and $P_{T}(q)$. It is interesting to note that for polymer blends $S_{0 \mathrm{AB}}(q)=0$ and we recover the well-known RPA result:

$$
S^{-1}(q)=S_{0 \mathrm{~A}}^{-1}(q)+S_{0 \mathrm{~B}}^{-1}(q)-2 \chi_{\mathrm{AB}}
$$

from which the spinodal line for macrophase separation defined by (2) would be readily obtained at $q=0$.

To describe the lamellar structure in Figure 3, one may use the free energy expression that has two contributions, one from the interfacial tension $\gamma_{\mathrm{AB}}$ at the $\mathrm{AB}$ interface and the other from the entropy loss due to the ordered structure:

$$
\frac{f_{\mathrm{Lam}}}{k_{\mathrm{B}} T}=\frac{3}{2}\left[\frac{d^{2}}{N l^{2}}-1\right]+\gamma_{\mathrm{AB}} \sigma-N \chi_{\mathrm{AB}} \phi_{\mathrm{A}} \phi_{\mathrm{B}},
$$

$\sigma$ being the surface area per chain. Minimization of this expression with respect to $d$ gives the characteristic length for microphase separation of the lamellar structure.

In the presence of nanoparticles such as PCBM, this theory can be generalized to identify structural changes, novel morphology developments, and phase properties. We are currently undergoing investigations along these lines, and we hope to report on some new results in the near future [38]. We may point out that, under certain conditions, the nanoparticles become preferentially solubilized by one of the blocks, say B as illustrated in Figure 3(b). Then one can show that the structure factor $S(q)$ satisfies a similar formula as in (3) with a renormalized apparent interaction parameter $\chi_{\text {app }}$ that depends on the properties of the nanoparticles.

On the other hand, a particular attention is given in the literature to the case of diblock copolymers where one block is rigid and the other is flexible (see Figure 3(c)).

\section{Structure Formation in Rod-Coil Block Copolymer Systems}

In the discussion above it has been assumed that the block copolymer has the characteristics of flexible ideal chains. In contrast, conductive block copolymers-by virtue of their chemical constitution that includes conjugated double bonds-represent rod-coil type character that is expected to result in deviations from the phase behavior discussed above. While there have been no studies on rod-coil type block copolymer/nanoparticle blend materials, rod-coil type architectures have received significant attention due to the relevance of liquid crystalline materials. This section summarizes the role of rod-coil type chain characteristics on the structure formation in block copolymer materials. Future research will have to elucidate the effect of chain stiffness on the miscibility and structure formation in particle blend materials. As elaborated above, an ideal morphology corresponds to cases where the size of concomitant $\mathrm{D}$ and $\mathrm{A}$ domains is below the mean diffusion length of excitons and 
direct pathways to the electrodes are available for charge collection. The mobility of charges should be sufficiently high to avoid losses. Morphologies exhibiting donor phases surrounded by acceptor phases represent a major challenge of research in polymer-based photovoltaic devices. This is why intense efforts to seek an optimal morphology satisfying those requirements are currently made focusing on interpenetrated polymer networks and block copolymers. Ordered phases favor the mobility of charges compared to amorphous ones, which means that the morphology can be tuned to provide ways for improving the efficiency of polymer-based photovoltaic cells.

Morphology is sensitive to the conditions of preparation of the polymer medium and has an impact on different steps of the conversion process starting from the exciton diffusion to its dissociation and the flow of charges to the electrodes. The method used to deposit polymer films on electrodes and thermal treatment after polymer deposit, the nature of solvent, its rate of evaporation, and solubility are crucial aspects in the elaboration process. The polymer crystalline structure, the conformation of chains, and the proximity of $\mathrm{D}$ and $\mathrm{A}$ domains throughout the active medium are equally important considerations [39-42]. The nature of blend components (electronics A and D polymers) and the method of preparation both enter in the optimization of morphology and the formation of bi-continuous phases with nanosized domains of tunable dimensions. Diblock copolymers offer the possibility to have A and D blocks within the same chain since a covalent bond forces them to remain tied together. One only needs to choose properly the nature of comonomers and chain architecture to achieve the desired morphology yielding the optimal conversion efficiency and the right optoelectronic responses. Therefore, a good selection of the chemical components that exhibit the proper electronic functions and permit tuning of the morphology to autoassembled structures constitutes the strategy to be adopted for enhancing the performances of polymer-based photovoltaic cells. Rod-coil diblock copolymers were found to exhibit good properties in terms of absorption of light, charge transport, tunable nanostructure, and morphology at the molecular level $[16,43,44]$. In such diblocks, a subtle competition arises between liquid-crystalline ordering driven by the rigid part and thermodynamic microphase separation due to the $\chi$-parameter. A proper choice of the flexible part is important to generate enough mobility and favor developments of the self-organization process $[45,46]$. A good control of the morphology with nanoassembled structures and crystalline orders insuring thermodynamic stability is the strategy to go for enhancing the efficiency on the long run. These systems lead to a significant enhancement of charge mobility and conversion efficiencies [47]. Their phase diagrams exhibit different ordered structures due to the ability of the rigid block to undergo an orientational nematic order either by a change of temperature or by an increase in the concentration. A theoretical scheme often used to describe the transition from an isotropic state to a nematic order is the Maier-Saupe theory [48] supplementing the Flory-Huggins theory of isotropic mixing. Such a diblock rigid flexible copolymer may also exhibit a smectic order with a lamellar structure of thickness $d$. Work is in progress to deal with those aspects in relation with the morphology, phase behavior, and structural properties of diblock copolymers systems in an effect to enhance the efficiency of polymerbased photovoltaic solar cells.

\section{Conclusion}

It is commonly accepted that the development of polymerbased photovoltaic technologies has potential to significantly add to a more sustainable use of energy resources and to facilitate a range of new technologies that will benefit from the economic viability of synthesis and processing of polymer materials. Block copolymers present an ideal platform to facilitate thermodynamic stable bulk heterojunction materials that present a major challenge in current polymer photovoltaic designs. Barriers that impede the use of block copolymers in current applications encompass the absence of suitable electron-conducting polymer systems (for allpolymer photoactive materials) as well as a lack of understanding of the governing parameters that control structure formation in block copolymer blend materials. Important information relating to these governing parameters can be deduced from recent work in related areas of polymer science. This paper has surveyed some of the major insights gained from the study of rodlike block copolymer as well as block copolymer/nanoparticle blend systems that bear relevance to potential bulk heterojunction designs. The authors do not see fundamental barriers in applying similar material concepts to bulk heterojunctions provided that the synthesis of appropriate semiconducting block copolymers can be accomplished. Previous work (on block copolymer/nanoparticle blends) suggests that enthalpic neutralization of PCBM fillers to block copolymers comprising a semiconducting block (such as P3HT) and amorphous polymers (such as PS) should facilitate efficient segregation of the filler particles to the interface in microphase-separated structures. To successfully realize this approach research should focus on the better understanding of the thermodynamic parameters involved in PCBM polymer blends and the development of efficient modification techniques for PCBM (e.g., with random copolymers comprised of $\mathrm{P} 3 \mathrm{HT}$ and PS to allow for neutralization) as well as the better understanding of the phase behavior of rod-coil block copolymer systems. Once established, diblock copolymers would be particularly suitable for enhancing anisotropy within the microstructures by a variety of techniques such as mechanical field alignment and roll casting. Such techniques could be used to facilitate large area alignment of the microstructure and significantly reduce the number of defects that present a big challenge in neat electronic polymer systems [42-55]. The use of diblock copolymers in which donor is a good hole conductor (typically P3HT-type) and the other block is aliphatic (such as PE) can be envisaged. The "nonelectronic block" helps to reduce oxygen diffusion enhancing the stability and lifetime of PV devices (electronic polymers are generally very sensitive to degradation by oxidative environments). This aspect is crucial for practical significance. Block copolymers of the type PE-P3HT (or 
similar) could also be of interest if the electron-conducting species (such as PCBM) is sequestered within the nonelectronic block. This would lead to a "thermodynamically stable" analogue to the classical bulk heterojunction geometry. The segregation of "particle-type" fillers in block copolymer domains has been extensively studied in the case of block copolymer/nanoparticle blend systems, and similar strategies could work for electronic/nonelectronic block copolymer systems.

\section{References}

[1] R. H. Bube, Photovoltaic Materials, Imperial College Press, London, UK, 1998.

[2] S. S. Sun and N. S. Sariciftci, Eds., Organic Photovoltaics: Mechanisms, Materials, and Devices, CRC Press, Boca Raton, Fla, USA, 2005.

[3] B. C. Thompson and J. M. Fréchet, "Polymer-fullerene composite solar cells," Angewandte Chemie International Edition, vol. 47, no. 1, pp. 58-77, 2008.

[4] J. M. Nunzi, "Organic photovoltaic materials and devices," Comptes Rendus Physique, vol. 3, no. 4, pp. 523-342, 2008.

[5] S. Günes, H. Neugebauer, and N. S. Sariciftci, "Conjugated polymer-based organic solar cells," Chemical Reviews, vol. 107, no. 4, pp. 1324-1338, 2007.

[6] T. S. Huang, C. Y. Huang, Y. K. Su et al., "High-efficiency polymer photovoltaic devices with glycerol-modified buffer layer," IEEE Photonics Technology Letters, vol. 20, no. 23, pp. 1935-1937, 2008.

[7] W. Wang, H. Wu, C. Yang et al., "High-efficiency polymer photovoltaic devices from regioregular-poly(3-hexylthiophene-2,5diyl) and [6,6]-phenyl- $\mathrm{C}_{61}$-butyric acid methyl ester processed with oleic acid surfactant," Applied Physics Letters, vol. 90, no. 18, Article ID 183512, 2007.

[8] M. R. Reyes, K. Kim, and D. L. Carroll, "High-efficiency photovoltaic devices based on annealed poly(3-hexylthiophene) and 1-(3-methoxycarbonyl)-propyl-1- phenyl- $(6,6) \mathrm{C}_{61}$ blends," Applied Physics Letters, vol. 87, no. 8, Article ID 083506, 3 pages, 2005.

[9] W.-L Ma, C. Y. Yang, X. Gong, K. Lee, and A. J. Heeger, "Thermally stable, efficient polymer solar cells with nanoscale control of the interpenetrating network morphology," Advanced Functional Materials, vol. 15, no. 10, pp. 1617-1622, 2005.

[10] R. A. Segalman, C. Brochon, and G. Hadziioannou, "Solar Cells Based on Diblock Copolymers: a PPV donor block and a fullerene derivatized acceptor block," in Organic Photovoltaics: Mechanisms, Materials, and Devices, S.-S. Sun and N. S. Sariciftci, Eds., CRC Press, Boca Raton, Fla, USA, 2005.

[11] J. L. Brédas and R. Silbey, "Excitons surf along conjugated polymer chains," Science, vol. 323, pp. 348-349, 2009.

[12] E. Collini and G. D. Scholes, "Coherent intrachain energy migration in a conjugated polymer at room temperature," Science, vol. 323, no. 5912, pp. 369-373, 2009.

[13] R. A. Segalman, B. McCulloch, S. Kirmayer, and J. J. Urban, "Block copolymers for organic optoelectronics," Macromolecules, vol. 42, no. 23, pp. 9205-9216, 2009.

[14] J. A. Malen, P. Doak, K. Baheti, T. D. Tilley, R. A. Segalman, and A. Majumdar, "The nature of transport variations in molecular heterojunction electronics," Nano Letters, vol. 9, no. 10, pp. 3406-3412, 2009.
[15] L. J. Lindgren, F. Zhang, M. Andersson et al., "Synthesis, characterization, and devices of a series of alternating copolymers for solar cells," Chemistry of Materials, vol. 21, no. 15, pp. 3491-3502, 2009.

[16] M. Sommer, S. M. Lindner, and M. Thelakkat, "Microphaseseparated donor-acceptor diblock copolymers: influence of HOMO energy levels and morphology on polymer solar cells," Advanced Functional Materials, vol. 17, no. 9, pp. 1493-1500, 2007.

[17] S. G. Cloutier, "Hybrid polyfluorene-based optoelectronic devices," in Advanced Photonic Sciences, M. Fadhali, Ed., In Tech, 2012.

[18] M. T. Dang, L. Hirsch, and G. Want, "P3HT:PCBM, best seller in polymer photovoltaic research," Advanced Materials, vol. 23, no. 31, pp. 3597-3602, 2011.

[19] D. Chen, A. Nakahara, D. Wei, D. Nordlund, and T. P. Russell, "P3HT/PCBM bulk heterojunction organic photovoltaics: correlating efficiency and morphology," Nano Letters, vol. 11, no. 2, pp. 561-567, 2011.

[20] J. Szmytkowski, "Modeling the electrical characteristics of P3HT:PCBM bulk heterojunction solar cells: implementing the interface recombination," Semiconductor Science and Technology, vol. 25, no. 1, Article ID 015009, 2010.

[21] M. R. Bockstaller, R. A. Mickiewicz, and E. L. Thomas, "Block copolymer nanocomposites: perspectives for tailored functional materials," Advanced Materials, vol. 17, no. 11, pp. 1331-1349, 2005.

[22] L. Leibler, "Theory of microphase separation in block copolymers," Macromolecules, vol. 13, no. 6, pp. 1602-1617, 1980.

[23] F. S. Bates and G. H. Fredrickson, "Block copolymer thermodynamics: theory and experiment," Annual Review of Physical Chemistry, vol. 41, no. 1, pp. 525-557, 1990.

[24] F. Holger, "Block copolymer phases of diblock, triblock and miktoarm star copolymers," can be found under, http://lmom.epfl.ch/courses/class_supramolecular_24.pdf.

[25] I. W. Hamley, Introduction to Block Copolymers, John Wiley \& Sons, 2004.

[26] U. Mitschke and P. Bäuerle, "The electroluminescence of organic materials," Journal of Materials Chemistry, vol. 10, pp. 1471-1507, 2000.

[27] R. E. Martin, F. Geneste, and A. B. Holmes, "Synthesis of conjugated polymers for application in light-emitting diodes (PLEDs)," Comptes Rendus de l'Académie des Sciences Paris 1, pp. 447-470, 2000.

[28] R. B. Thompson, V. V. Ginzburg, M. W. Matsen, and A. C. Balazs, "Predicting the mesophases of copolymer-nanoparticle composites," Science, vol. 292, pp. 2469-2472, 2001.

[29] H. Tanaka, H. Hasegawa, and T. Hashimoto, "Ordered structure in mixtures of a block copolymer and homopolymers. 1. Solubilization of low molecular weight homopolymers," Macromolecules, vol. 24, no. 1, pp. 240-251, 1991.

[30] K. I. Winey, E. L. Thomas, and L. J. Fetters, "Swelling a lamellar diblock copolymer with homopolymer: influences of homopolymer concentration and molecular weight," Macromolecules, vol. 24, no. 23, pp. 6182-6188, 1991.

[31] J. J. Chiu, B. J. Kim, E. J. Kramer, and D. J. Pine, "Control of nanoparticle location in block copolymers," Journal of the American Chemical Society, vol. 127, pp. 5036-5037, 2005.

[32] J. Listak, I. F. Hakem, H. J. Ryu et al., "Effect of chain architecture on the compatibility of block copolymer/nanoparticle blends," Macromolecules, vol. 42, no. 15, pp. 5766-5773, 2009. 
[33] Y. Zhang, K. Tajima, and K. Hashimoto, "Nanostructure formation in poly(3-hexylthiophene-block- 3-(2-ethylhexyl)thiophene)s," Macromolecules, vol. 42, no. 18, pp. 7008-7015, 2009.

[34] Y. C. Tseng and S. B. Darling, "Block copolymer nanostructures for technology," Polymers, vol. 2, no. 4, pp. 470-489, 2010.

[35] B. D. Olsen and R. A. Segalman, "Structure and thermodynamics of weakly segregated rod-coil block copolymers," Macromolecules, vol. 38, no. 24, pp. 10127-10137, 2005.

[36] P. J. Flory, Introduction to Polymer Chemistry, Cornell University Press Ithaca, 1956.

[37] P. J. de Gennes, Basic Concepts in Polymer Physics, Cornell University Press Ithaca, 1979.

[38] I. F. Hakem, M. R. Bockstaller, A. Benmouna, R. Benmouna, and M. Benmouna, "Structural characterization of iron oxide nanoparticles doped norbornene-norbornene dicarboxylic acid diblock copolymers: theory versus experiments," in preparation.

[39] R. Duan, L. Ye, X. Guo et al., "Application of two-dimensional conjugated benzo[1,2-b:4,5-b']dithiophene in QuinoxalineBased Photovoltaic Polymers," Macromolecules, vol. 45, no. 7, pp. 3032-3038, 2012.

[40] M. T. Rispens, A. Meetsma, R. Rittberger, C. J. Brabec, N. S. Sariciftci, and J. C. Hummelen, "Influence of the solvent on the crystal structure of PCBM and the efficiency of MDMOPPV:PCBM "plastic" solar cells," Chemical Communications, vol. 9, no. 17, pp. 2116-2118, 2003.

[41] Y. Kim, S. Cook, S. M. Tuladhar et al., "A strong regioregularity effect in self-organizing conjugated polymer films and highefficiency polythiophene: fullerene solar cells," Nature Materials, vol. 5, no. 3, pp. 197-203, 2006.

[42] R. Bechara, N. Leclerc, P. Lévêque, F. Richard, T. Heiser, and G. Hadziioannou, "Efficiency enhancement of polymer photovoltaic devices using thieno-thiophene based copolymers as nucleating agents for polythiophene crystallization," Applied Physics Letters, vol. 93, no. 1, Article ID 013306, 3 pages, 2008.

[43] S. M. Lindner and M. Thelakkat, "Nanostructures of n-type organic semiconductor in a p-type matrix via self-assembly of block copolymers," Macromolecules, vol. 37, no. 24, pp. 88328835, 2004.

[44] S. M. Lindner, S. Hüttner, A. Chiche, M. Thelakkat, and G. Krausch, "Charge separation at self-assembled nanostructured bulk interface in block copolymers," Angewandte Chemie, vol. 45, no. 20, pp. 3364-3368, 2006.

[45] N. Sary, R. Mezzenga, C. Brochon, G. Hadziioannou, and J. Ruokolainen, "Weakly segregated smectic C lamellar clusters in blends of rods and rod-coil block copolymers," Macromolecules, vol. 40, no. 9, pp. 3277-3286, 2007.

[46] M. C. Iovu, C. Rockford Craley, E. M. Jeffries et al., "Conducting regioregular polythiophene block copolymer nanofibrils synthesized by reversible addition fragmentation chain transfer polymerization (RAFT) and Nitroxide Mediated Polymerization (NMP)," Macromolecules, vol. 40, no. 14, pp. 4733-4735, 2007.

[47] V. Gernigon, P. Lévêque, C. Brochon et al., "Fullerene-grafted block copolymers used as compatibilizer in P3HT/PCBM bulk heterojunctions: morphology and photovoltaic performances," The European Physical Journal, vol. 56, no. 3, pp. 34107-34112, 2011.

[48] F. Brochard, J. Jouffroy, and P. Levinson, "Phase diagrams of mesomorphic mixtures," Journal de Physique Paris, vol. 45, no. 7, pp. 1125-1136, 1984.
[49] R. A. Segalman, B. McCulloch, S. Kirmayer, and J. J. Urban, "Block copolymers for organic optoelectronics," Macromolecules, vol. 42, no. 23, pp. 9205-9216, 2009.

[50] A. M. Rosales, B. L. McCulloch, R. N. Zuckermann, and R. A. Segalman, "Tunable phase behavior of polystyrene-polypeptoid block copolymers," Macromolecules, vol. 45, no. 15, pp. 60276035, 2012.

[51] M. A. Villar, D. R. Rueda, F. Ania, and E. L. Thomas, "Study of oriented block copolymers films obtained by roll-casting," Polymer, vol. 43, no. 19, pp. 5139-5145, 2002.

[52] J. W. Park and E. L. Thomas, "Multiple ordering transitions: hierarchical self-assembly of rod-coil block copolymers," Advanced Materials, vol. 15, no. 78, pp. 585-588, 2003.

[53] J. A. Odell, A. Keller, E. D. T. Atkins, and M. J. Miles, "Structural studies of poly(p-phenylene benzbisthiazole) films," Journal of Materials Science, vol. 16, no. 12, pp. 3309-3318, 1981.

[54] M. C. Iovu, R. Zhang, J. R. Cooper et al., "Conducting block copolymers of regioregular poly(3-hexylthiophene) and poly(methacrylates): electronic materials with variable conductivities and degrees of interfibrillar order," Macromolecular Rapid Communications, vol. 28, no. 17, pp. 1816-1824, 2007.

[55] G. Zotti, B. Vercelli, A. Berlin et al., "Self-assembled structures of semiconductor nanocrystals and polymers for photovoltaics. 2. Multilayers of CdSe nanocrystals and oligo(poly)thiophenebased molecules. Optical, electrochemical, photoelectrochemical, and photoconductive properties," Chemistry of Materials, vol. 22, no. 4, pp. 1521-1532, 2010. 

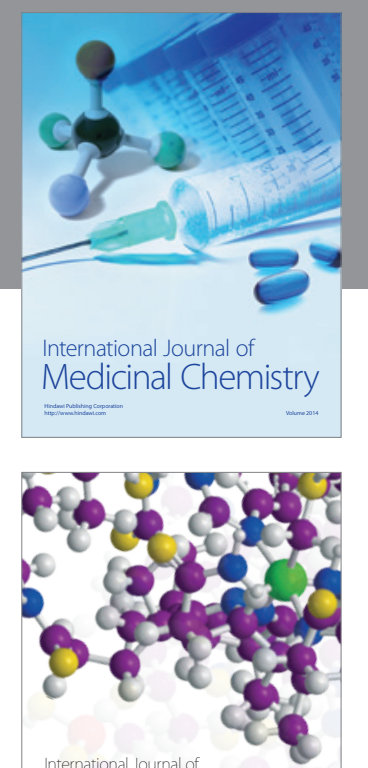

\section{Carbohydrate} Chemistry

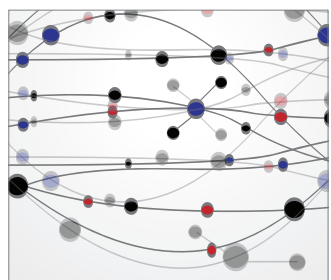

The Scientific World Journal
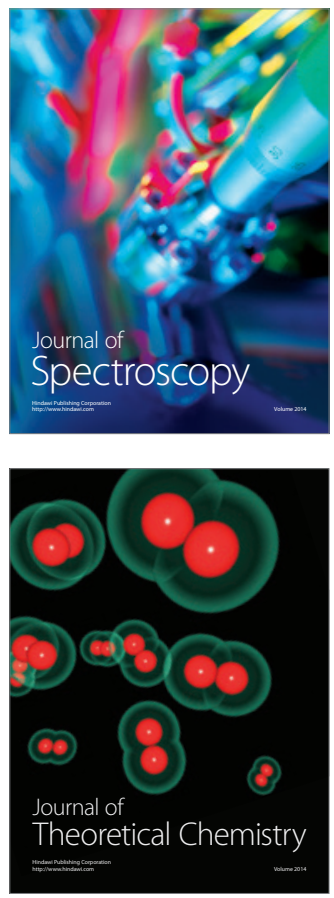
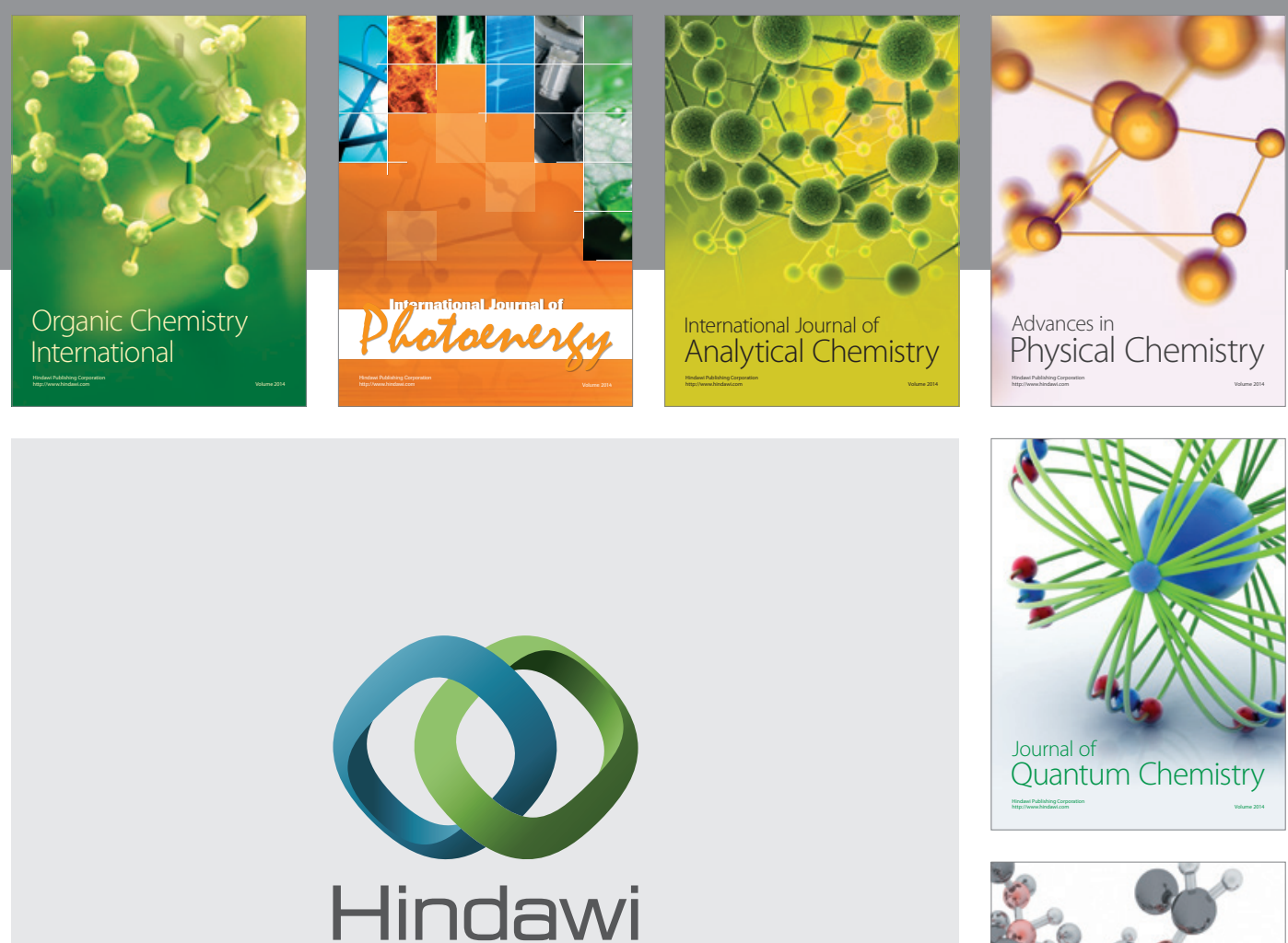

Submit your manuscripts at

http://www.hindawi.com

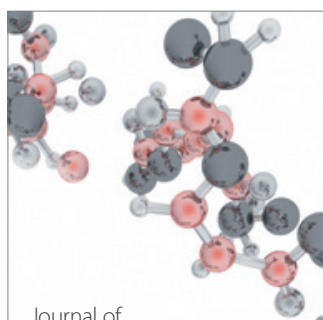

Analytical Methods

in Chemistry

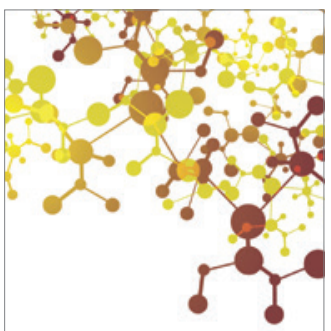

Journal of

Applied Chemistry

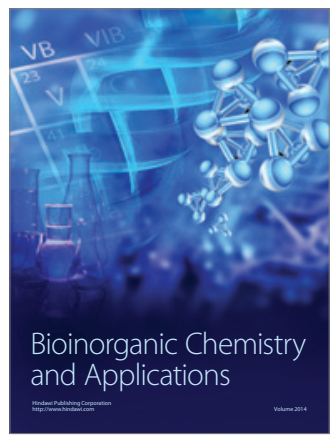

Inorganic Chemistry
